\title{
The NASA X-ray Mission Concepts Study
}

R. Petre*a, A. Ptak ${ }^{\mathrm{a}}$, J. Bookbinder ${ }^{\mathrm{b}}$, M. Garcia ${ }^{\mathrm{b}}$, R. Smith ${ }^{\mathrm{b}}$, M. Bautz ${ }^{\mathrm{c}}$, J. Bregman ${ }^{\mathrm{d}}$, D. Burrows ${ }^{\mathrm{e}}$, W. Cash ${ }^{f}$, C. Jones-Forman ${ }^{b}$, S. Murray ${ }^{g}$, P. Plucinsky ${ }^{b}$, B. Ramsey ${ }^{\text {h }}$, R. Remillard ${ }^{b}$, C. WilsonHodge $^{\text {h }}$, G. Daelemans ${ }^{\text {a }}$, G. Karpati ${ }^{a}$, A. Nicoletti ${ }^{a}$, P Reid ${ }^{b}$

aNASA / GSFC, Greenbelt, MD 20771, USA; 'Harvard-Smithsonian Center for Astrophysics, Cambridge, MA 01238, USA; MIT Center for Space Research, Cambridge, MA 02139, USA; Department of Astronomy, University of Michigan, Ann Arbor, MI 48109, USA; Department of Astronomy and Astrophysics, Pennsylvania State University, University Park, PA 16802, USA; CASA, University of Colorado, Boulder, CO 80309, USA; Department of Physics and Astronomy, Johns Hopkins University, Baltimore, MD 21218, USA; NASA / MSFC, Huntsville, AL 35812, USA

\begin{abstract}
The 2010 Astrophysics Decadal Survey recommended a significant technology development program towards realizing the scientific goals of the International X-ray Observatory $(L X O)$. NASA has undertaken an X-ray mission concepts study to determine alternative approaches to accomplishing $I X O$ 's high ranking scientific objectives over the next decade given the budget realities, which make a flagship mission challenging to implement. The goal of the study is to determine the degree to which missions in various cost ranges from $\$ 300 \mathrm{M}$ to $\$ 2 \mathrm{~B}$ could fulfill these objectives. The study process involved several steps. NASA released a Request for Information in October 2011, seeking mission concepts and enabling technology ideas from the community. The responses included a total of 14 mission concepts and 13 enabling technologies. NASA also solicited membership for and selected a Community Science Team (CST) to guide the process. A workshop was held in December 2011 in which the mission concepts and technology were presented and discussed. Based on the RFI responses and the workshop, the CST then chose a small group of notional mission concepts, representing a range of cost points, for further study. These notional missions concepts were developed through mission design laboratory activities in early 2012. The results of all these activities were captured in the final Xray mission concepts study report, submitted to NASA in July 2012. In this presentation, we summarize the outcome of the study. We discuss background, methodology, the notional missions, and the conclusions of the study report.
\end{abstract}

Keywords: International X-ray Observatory, X-ray astronomy, mission concepts

\section{INTRODUCTION}

The International $X$-ray Observatory $(I X O)$ was a joint NASA/ESA/JAXA mission designed to address major astrophysics questions, including: "What happens close to a black hole?"; "How and when did supermassive black holes grow?"; "How does large scale structure evolve?"; "What is the connection between supermassive black holes and large scale structure in the Universe?"; and "How does matter behave at high density?" IXO addressed these questions through a combination of a very large area, high-resolution X-ray mirror and state of the art instrumentation, including a microcalorimeter, grating spectrometer and wide field imager. ${ }^{1-2}$

In "New Worlds, New Horizons (NWNH)," the 2010 report of the Decadal Survey of Astronomy and Astrophysics, IXO was described as " $a$ versatile, large-area, high-spectral-resolution $X$-ray telescope that will make great advances on broad fronts ranging from characterization of black holes to elucidation of cosmology and the life cycles of matter and energy in the cosmos." ${ }^{3}$ Despite the importance of the $I X O$ science, the mission was ranked fourth among large NASA missions for the next decade because of its projected cost.

IXO had also been proposed to ESA as a candidate for the L-1 opportunity in its Cosmic Visions program. Following

"robert.petre-1@nasa.gov phone 1 301-286-3844

Updated 1 March 2012 
the release of $N W N H$, the Planetary Science Decadal Survey, and the U.S. budgets in February 2011, ESA announced revised plans for all three of the L-class missions under study (IXO, LISA, EJSM), restructuring them into ESA-only missions. This resulted in the development of ATHENA and the termination of the IXO program.

Recognizing the importance of the $I X O$ scientific objectives in the absence of a defined mission, NASA commissioned a study to identify approaches to fulfilling them at a cost more consistent with the available resources over the next decade. The primary study activity consisted of defining a set of notional missions and estimating their cost. This paper briefly describes the study methodology, the notional missions, and the overall study conclusions.

\section{THE STUDY PROCESS}

The study was initiated by two solicitations from NASA Headquarters. The first was a Request for Information (RFI) "seeking information that can be used to develop concepts that meet some or all of the scientific objectives of the International X-ray Observatory $(\mathrm{IXO})$." The input that was sought included "relevant mission concepts, instrument concepts, enabling technologies, or any aspect of flight, ground or launch systems architecture." The RFI was released on 13 September 2011 with responses due on 28 October 2011. In parallel, NASA invited the astronomy community to apply for membership on a Community Science Team (CST), whose function was to review all RFI responses and define a set of mission concepts for further study. These concepts were to represent various cost points between $\$ 300 \mathrm{M}$ to $\$ 2,0$ COM. NASA selected ten CST members, who; along with the Study Manager, Study Scientist, and the Science and Engireering Support Teams, composed the X-ray Mission Concepts Study Team. The Study Team members are listed in Table 1.

\section{Table 1: X-ray Mission Concepts Study Team}

\begin{tabular}{|l|l|}
\hline Stady Manager & Gerry Daelemans (GSFC) \\
\hline Study Scientist & Rob Petre (GSFC) \\
\hline $\begin{array}{l}\text { Community Science } \\
\text { Team }\end{array}$ & $\begin{array}{l}\text { Joel Bregman (Chair - Michigan), Mark Bautz (MIT), David Burrows (Penn State), Webster } \\
\text { Cash (U Colorado), Christine Jones (SAO), Stephen Murray (JHU), Paul Plucinsky (SAO), } \\
\text { Brian Ramsey (MSFC), Ron Remillard (MIT), Colleen Wilson-Hodge (MSFC) }\end{array}$ \\
\hline Science Support Team & Andy Ptak (GSFC), Jay Bookbinder, Randall Smith, Michael Garcia (SAO) \\
\hline $\begin{array}{l}\text { Engineering Support } \\
\text { Team }\end{array}$ & $\begin{array}{l}\text { Tony Nicoletti, Gabe Karpati, Sharon Seipel (GSFC), Mark Freeman, Paul Reid (SAO), GSFC } \\
\text { MDL and IDL engineers }\end{array}$ \\
\hline
\end{tabular}

A total of 30 RFI responses were received. Submissions came from universities, industry, NASA centers, and federally funded research labs. Fourteen of these responses described mission concepts, one response described a program strategy, 12 responses described enabling technology, and three described instrument concepts.

The missions and instruments and their basic characteristics are summarized in Table 2. Many of the mission concepts emphasized high-resolution spectroscopy, six using an IXO-like X-ray calorimeter (AXSIO, EPE, SAHARA, SMART-X, $W F X I S$, and Xenia) and four using dispersive X-ray gratings (AEGIS, AXSIO, SMART-X, and WHIMex). Three emphasized wide field imaging (SMART-X, WFXT, and WFXIS), six emphasized hard X-rays (AXTAR, BEST, EREXS, $H E X-P, B H T$, and Xenia) and/or all sky monitoring (AXTAR, EREXS, SuperMon, and Xenia). One included a polarimeter (BEST).

Based on estimates provided in the RFI responses, the first three mission concepts listed in Table 2 fell into the "large" cost bin of $>\$ 1 \mathrm{~B}$ between $\sim \$ 600 \mathrm{M}$ and $\$ 1 \mathrm{~B}$. The next six mission concepts fell into the "medium" cost bin between $\$ 600 \mathrm{M}$ and $\$ 1 \mathrm{~B}$, and the final five fell into the "small" cost bin of $<\$ 600 \mathrm{M}$. The final two entries in Table 2 are instrument concepts. In general, the cost of a mission concept directly related to the degree to which it could address $I X O$ science goals. The most expensive, $\sim \$ 1-2 \mathrm{~B}$ class mission concepts, typically addressed the majority of the IXO science objectives. The medium class mission concepts addressed a substantial (but varying) fraction of the $I X O$ science 
objectives, on occasion addressing all of the topics to a greater or lesser degree. The smallest concepts typically addressed a single $I X O$ science area. Some of the measurement approaches duplicated those that would have been taken by $I X O$, while for others novel approaches were suggested.

Table 2. Instrument and Mission Concepts

\begin{tabular}{|c|c|c|c|c|c|}
\hline Mission & $\begin{array}{c}\text { Randpass } \\
\text { keV }\end{array}$ & $\begin{array}{l}\text { Effective Area } \\
\text { mºlekeV }^{2}\end{array}$ & $\begin{array}{l}\text { Field of View } \\
\text { arcmin }\end{array}$ & $\begin{array}{l}\text { Ang. Res. }{ }^{*} \\
\text { arcsec }\end{array}$ & Instrument(s) \\
\hline AXSIO & $0.2-10$ & $0.9 @ 1,0.2 @ 6$ & 4 & 10 & $\begin{array}{l}\text { Calorimeter, } \\
\text { Grating Spectrometer }\end{array}$ \\
\hline SMART-X & $0.2-10$ & 2.3@1,0.2@6 & 22 & 0.5 & $\begin{array}{l}\text { Calorimeter, } \\
\text { Grating Spectrometer, } \\
\text { Wide Field Imager }\end{array}$ \\
\hline Xenia & $0.2-5.0$ & $0.053 @ 1$ & 84 & 15 & $\begin{array}{l}\text { Cryogenic Imaging } \\
\text { Spectrometer, } \\
\text { High Angular Resolution } \\
\text { Imager, } \\
\text { Translent Event Detector }\end{array}$ \\
\hline AEGIS & $0.25-2.0$ & $0.14 @ 0.6$ & 19 & 10 & Grating Spectrometer \\
\hline$E P E$ & $0.3-10$ & $0.5 @ 1,0.2 @ 6$ & 8 & 60 & Calorimeter \\
\hline EREXS & $5-300$ & $0.7 @ 100$ & $70^{\circ}$ & 20 & $\begin{array}{l}\text { Hard X-ray Imager, Infrared } \\
\text { Telescope }\end{array}$ \\
\hline SAHARA & $0.2-3.0$ & $0.3 @ 1$ & 8 & 5 & Calorimeter \\
\hline WFXIS & $0.1-2.5$ & 0.04@1 & 15 & 10 & Calorimeter \\
\hline WFXT & $0.2-4.0$ & $0.7 @ 1$ & 60 & 5 & Wide Field Imager \\
\hline AXTAR & $2-30$ & 3.2@6 & 60 & 60 & $\begin{array}{l}\text { Large Area Timing Array, } \\
\text { All Sky Monitor }\end{array}$ \\
\hline BEST & 2-70 & $0.4 @ 2,0.3 @ 6$ & 12 & 10 & $\begin{array}{l}\text { Cadmium-Zinc-Telluride } \\
\text { array, } \\
\text { Polarimeter }\end{array}$ \\
\hline$H E X-P$ & $0.15-200$ & $\begin{array}{c}0.8 @ 6, \\
0.15 @ 50\end{array}$ & 13 & 15 & $\begin{array}{l}\text { Silicon + Cadmium-Telluride } \\
\text { array }\end{array}$ \\
\hline $\begin{array}{l}\text { SuperMon \& } \\
\text { BHT }\end{array}$ & $\begin{array}{c}2-60 \\
0.5-30\end{array}$ & $\begin{array}{c}3 \times 0.04 @ 10 \\
5 @ 10\end{array}$ & $\begin{array}{c}4 \pi \\
120\end{array}$ & $0.5^{\circ}$ & $\begin{array}{l}\text { Low Energy Proportional } \\
\text { Counters, Silicon+Cadmium- } \\
\text { Zinc-Telluride sandwich }\end{array}$ \\
\hline WHIMex & $0.2-0.8$ & $.025 @ 0.6$ & nja & 15 & Grating Spectrometer \\
\hline $\begin{array}{l}\text { Hard X-ray } \\
\text { Telescope }\end{array}$ & $10-70$ & $.025 @ 30$ & 12 & 22 & $\begin{array}{l}\text { Cadmium-Zinc-Telluride } \\
\text { array }\end{array}$ \\
\hline $\begin{array}{l}\text { Soft X-ray } \\
\text { Polarimeter }\end{array}$ & $0.2-0.8$ & $.03 @ 0.4$ & $\mathbf{n} / \mathbf{a}$ & n/a & $\begin{array}{l}\text { Grating Polarimeter with } \\
\text { Charge Coupled Device } \\
\text { readout }\end{array}$ \\
\hline
\end{tabular}

The colors here represent estimated mission costs: $>\$ 1 \mathrm{~B}$ (blue), $\$ 0.6 \mathrm{~B}-\$ 1.0 \mathrm{~B}$ (green) and $<\$ 0.6 \mathrm{~B}$ (pink). Rows that are white show instruments.

*Measured as HPD

Representatives from each of the RFI response teams were invited to present their ideas to the science community at a workshop 14-15 December 2011. Over 100 astronomers attended, including the CST. These presentations and the ensuing discussion were critical in the CST formulating the set of "notional" missions. A series of technology talks made the case that none of these missions can proceed without a robust program that supports the cutting-edge 
technologies these missions would use. Based on the RFI responses and the input at the workshop, the CST defined three single-instrument "notional missions" that combined characteristics of various mission concepts from the RFI responses. These notional missions are: a calorimeter spectrometer $(N-C A L)$, a gratings spectrometer $(N-X G S)$, and a wide-field imager ( $N$-WFn). In addition, the CST carried forward $A X S I O$ as its representative "large" mission. This decision was based on the fact that $A X S I O$ was conceived in direct response to the recommendations in $N W N H$, and because it had already undergone a feasibility and cost study using the same approach that was to be used for the notional missions, and thus could be directly compared with minimum additional effort. The key properties of the three single-instrument missions and AXSIO are summarized in Table 3.

Table 3: Notional Missions

\begin{tabular}{|c|c|c|c|c|c|c|c|c|}
\hline Mission & Energy range & $\Delta \mathbf{E}$ & $\begin{array}{c}\text { Effective Area } \\
\mathrm{m}^{2} @ \mathbf{k e V}\end{array}$ & $\begin{array}{l}\text { Ang. } \\
\text { Res.* }\end{array}$ & $\begin{array}{l}\text { Field of } \\
\text { view }\end{array}$ & $\begin{array}{l}\text { Focal } \\
\text { length }\end{array}$ & $\begin{array}{l}\text { Cost } \\
\text { Goal }\end{array}$ & $\begin{array}{l}\text { MDL } \\
\text { Cost }\end{array}$ \\
\hline N-CAL & $0.2-10 \mathrm{keV}$ & $\begin{array}{c}<3 \mathrm{eV} \text { (inner } \\
\text { pixels) }\end{array}$ & $\begin{array}{l}0.5 @ 1 \\
0.2 @ 6\end{array}$ & $10 "$ & $4 "$ & $9.5 \mathrm{~m}$ & $<\$ 1 B$ & $\$ 1.2$ B \\
\hline$N-X G S$ & $0.2-1.3 \mathrm{keV}$ & $\lambda / \Delta \lambda>3000$ & $0.05 @ 0.2-1.3$ & $10^{\prime \prime}$ & $\mathrm{n} / \mathrm{a}$ & $4 \mathrm{~m}$ & $\begin{array}{c}<600 \\
M\end{array}$ & $\$ 0.8 \mathrm{~B}$ \\
\hline AXSIO & $\begin{array}{c}0.2-10 \mathrm{keV} \\
\text { (XMS) } \\
0.2-1.5 \mathrm{keV} \\
\text { (XGS) }\end{array}$ & $\begin{array}{c}<3 \mathrm{eV} \\
\lambda / \Delta \lambda>3000\end{array}$ & $\begin{array}{c}0.93 @ 1.25 \\
0.2 @ 6 \\
0.1 @ 0.3-1\end{array}$ & $10 "$ & $4^{\prime}$ & $10 \mathrm{~m}$ & $<\$ 2 \mathrm{~B}$ & $\$ 1.5 \mathrm{~B}$ \\
\hline$N$-WFI & $0.2-10 \mathrm{keV}$ & $150 \mathrm{eV}$ & $\begin{array}{l}0.7 @ 1 \\
0.2 @ 6\end{array}$ & 7" & $>24^{\prime}$ & $6 \mathrm{~m}$ & $<\$ 1 B$ & $\$ 1.0 \mathrm{~B}$ \\
\hline
\end{tabular}

Measured in HPD

The notional missions, taken together, address nearly the full suite of $I X O$ science objectives (as does the $A X S I O$ concept). This is shown in Table 4, which gives an overview of the primary IXO science objectives that would be accomplished by the notional missions assuming three-year mission lifetimes. In this table, darker shading and lower numerical values correspond to more comprehensive fulfillment of an IXO objective. The value [1] signifies essentially all $I X O$ science can be achieved for this topic, [2] signifies that some of the IXO science can be achieved, and [3] signifies only a fraction of $I X O$ science can be achieved. In the case of [2], the full $\mathrm{LXO}$ science can be achieved through an extended mission, while in the case of [3], the loss is not recoverable, for example due to inadequate spectral resolution. While $I X O$ 's timing capability is partially retained by the $A X S I O$ and $N$-CAL missions, $I X O$ 's hard X-ray imaging and polarimetry capabilities are not provided by any of the notional missions.

\section{Costing Methodology}

Each notional mission was subjected to a one-week GSFC Mission Design Lab (MDL) session. The goal of this effort was to apply a uniform process to the development of an initial concept design that would realistically meet the performance requirements set by the study team (i.e., a point design). Using standard cost estimating tools (most notably PRICE-H) and the same costing personnel for all estimates, the MDL then estimated the total mission lifetime costs, which can be used to compare missions, and which represent current best estimates of mission costs. For the most complex of the instruments (the calorimeter), the GSFC Instrument Design Lab (IDL) was used to develop instrument concept costs that were then passed to the MDL. These labs arrive at instrument and mission costs using parametric models (where key parameters include mass and complexity), supplemented by bottoms-up estimates as appropriate.

To the greatest extent possible, the costing approach for each notional mission design was identical. Specifically, the missions are all designated as high priority, low risk missions, with high to medium technical complexity and high to medium cost, and a minimum lifetime requirement of three years. This translates to being considered a NASA "Class B" mission, which imposes requirements with respect to mission assurance that significantly affect cost. These include the degree to which redundant systems are used, the depth and extent of a qualification test program (engineering models, engineering test and qualification units) and the pedigree of electric, electronic and electromechanical (EEE) 
parts. After consideration of factors involved in selecting a mission orbit (low-Earth, high-Earth, L2, etc.) for each mission, the study team determined that the science objectives for all missions could be met if placed in a Sun-Earth L2 orbit. This was merely a sufficient and not a necessary condition, but the brevity of the study precluded detailed tradeoffs between other possible orbits. Each design therefore includes propulsion, telemetry, orbit determination, etc., subsystems necessary for the $\mathbf{L} 2$ orbit. The launch vehicles, while not the same for each mission (due to differing throw weight requirements), were selected based on achieving this orbit.

Table 4: Primary IXO Science Objectives Addressed by Notional Missions

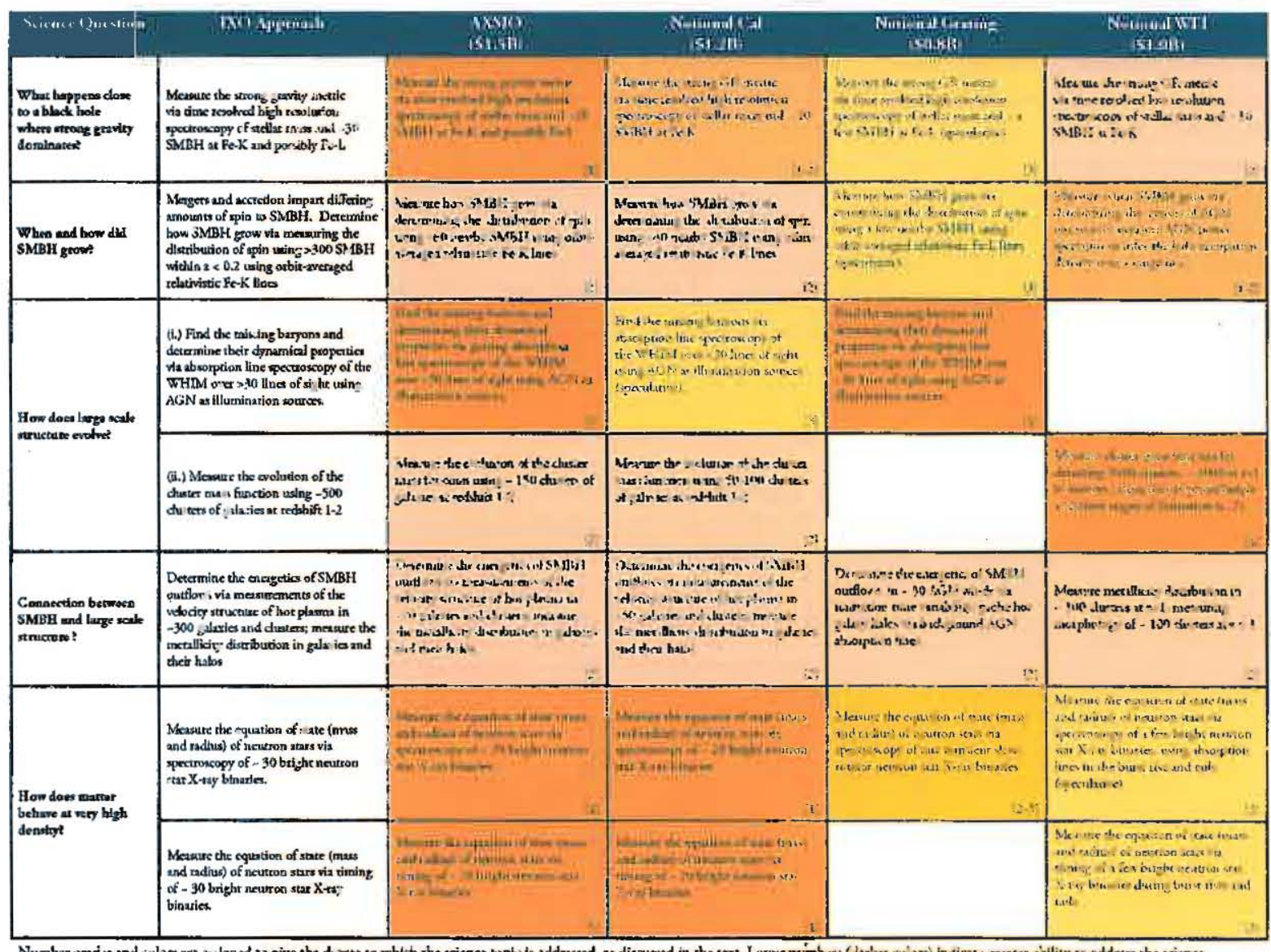

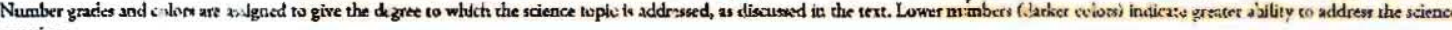
quesion.

For each notional mission, the PRICE-H cost modeler was used to cost the spacecraft based on the MDL design, instrument, and mirror. (The sole exception was the $N$-WFI mirror, which was a bottoms-up estimate.) For the costing of the instruments, mirrors, and spacecraft bus components, a minimum of TRL-6 readiness was assumed. Standard "wrap" factors were applied to estimate cost for Project Management, Mission Systems Engineering, Safety and Mission Assurance, Mission Level Integration and Test and Education and Public Outreach. Costs for Science, Phase E Mission Operations, and Ground Systems were based on experience gained during $I X O$ pre-phase A activities, and were scaled for each mission accordingly, accounting for data volumes, mission operation complexity, etc. The Mirror Facilities costs were also based on experience gained during $I X O$ pre-phase $\mathrm{A}$ activities.

Cost reserves of 30 percent were added to the instrument and mirrors, spacecraft bus, mission operations and ground data systems as well as the wrap factors. Mission Science had 10 percent cost reserves applied, while Education and Public Outreach, Launch Vehicle, and Science Grants, each had no cost reserves applied.

A critical assumption is that NASA is responsible for all mission costs. Considerable savings to NASA could be 
realized through partnership with other agencies (e.g., ESA, JAXA), allowing major hardware contributions.

\section{THE NOTIONAL MISSIONS}

\section{Notional Calorimeter Mission (N-CAL)}

The calorimeter-only notional mission $N-C A L$ is envisioned as a $\sim \$ 1 \mathrm{~B}$ class mission. Its key performance requirements include effective area of $5,000 \mathrm{~cm}^{2}$ at $1 \mathrm{keV}$ and $2,000 \mathrm{~cm}^{2}$ at $6 \mathrm{keV}$, a field of view of at least 4 arcmin, angular resolution of $10 \operatorname{arcsec}$ (HPD) or better, and absolute time resolution requirement of $1 \mathrm{~ms}$ with a goal of $100 \mu \mathrm{s}$. Such an imaging high-resolution spectrometer would directly address all five of the high-priority science goals of $I X O$, as identified by $N W N H$, to varying levels of fidelity, and address the $I X O$ science objectives more comprehensively than the other two single-instrument notional missions (see Table 4). Specifically, time resolved, high-resolution spectra of the relativistically broadened $\mathrm{Fe}-\mathrm{K}$ line in stellar mass or supermassive black holes would address the $I X O$ science objective "What happens close to a black hole?" Measurements of the mass and composition of clusters of galaxies at redshift $<2$ through spatially-resolved spectroscopy would address the IXO science objective "How does large scale structure evolve?" Finally, measurements of the metallicity and velocity structure of hot gas in galaxies and clusters with highresolution spectra would address the $I X O$ science objective "What is the connection between supermassive black hole formation and evolution of large scale structure (i.e., cosmic feedback)?" The high spectral resolution of the calorimeter, with spectra of sufficient statistical precision, is the key enabling capability for all of these science objectives.

\subsection{1 $N-C A L$ instrumentation}

The flight mirror assembly (FMA) utilizes a segmented design with precision slumped glass mirror segments. It has a focal length of $9.5 \mathrm{~m}$, a diameter of $1.3 \mathrm{~m}, 178$ shells, $20 \mathrm{~cm}$ segment length, and a mass of $325 \mathrm{~kg}$. The combined effective area of the FMA and the calorimeter, plotted in Fig. 1, provides more than an order of magnitude increase over the effective area of the Astro- $H$ Soft X-ray Spectrometer. In addition, the FMA also provides an order-of-magnitude improvement in angular resolution compared to Astro- $H(\sim 1.0$ arcmin HPD).

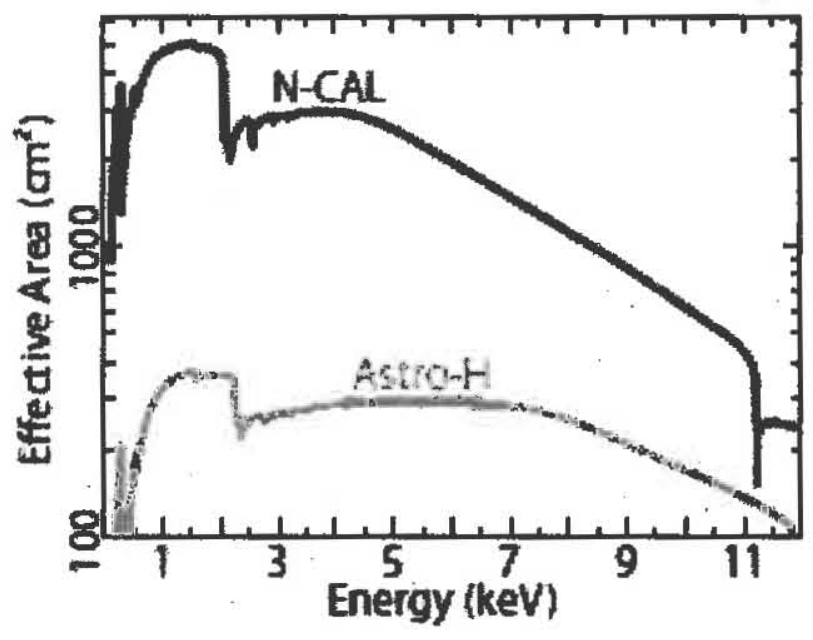

Figure 1: Effective Area of notional XMS mission compared to the calorimeter on Astro-H.

The calorimeter design would be a hybrid array consisting of an inner point-source array (PSA) consisting of smaller pixels, with higher spectral resolution and faster readout, and an outer array with larger pixels, slower multiplexed readouts and lower spectral resolution. The PSA would consist of $16 \AA \sim 16$ pixels with a spectral resolution of $2 \mathrm{eV}$ covering a FOV of $0.4 \times 0.4$ arcmin (each pixel is $1.5 \times 1.5 \mathrm{arcsec}$ ), requiring 256 Transition Edge Sensors (TESs) for readout. This improvement in the calorimeter configuration enables high count rate $(15,000 \mathrm{cps}$, or $100 \mathrm{mcrab})$, high spectral resolution $(2 \mathrm{eV})$ science, without the extra detector (HTRS) that was used on IXO. 
The outer array would complete the coverage of the $4 \times 4$ arcmin FOV with two types of pixels, each with a size of $6 \times 6$ arcsec. There would be 544 pixels each with its own dedicated TES to provide $3 \mathrm{eV}$ energy resolution surrounding the PSA. The outermost part of the array would be populated by 1,040 pixels with $6 \mathrm{eV}$ resolution. A single TES would provide the readout for four pixels, reducing the number of TESs required for this part of the array to 260 . The total number of TESs (and hence individual signal lines) for the instrument would be 1,060. Tables 3 and 5 summarize the relevant parameters of the calorimeter instrument envisioned for this mission.

Table 5: N-Cal Calorimeter Array Details

\begin{tabular}{|l|c|c|c|c|c|}
\hline \multicolumn{1}{|c|}{ Array } & FOV & \# Pixels & Pixel Slze & Resolution & \# TESs \\
\hline Inner PSA & $0.16 \mathrm{arcmin}^{2}$ & 256 & $1.5 \times 1.5^{\prime \prime}$ & $2 \mathrm{eV}$ & 256 \\
\hline Outer \#1 & $5.5 \mathrm{arcmin}^{2}$ & 544 & $6.0 \times 6.0^{\prime \prime}$ & $3 \mathrm{eV}$ & 544 \\
\hline Outer \#2 & $10.3 \mathrm{arcmin}^{2}$ & 1040 & $6.0 \times 6.0 "$ & $6 \mathrm{eV}$ & 260 \\
\hline
\end{tabular}

\subsubsection{N-CAL Mission Design and cost estimate}

An illustration of the N-CAL spacecraft design is shown in Fig. 2. The design produced by the MDL has a payload mass, including contingency, of $1775 \mathrm{~kg}$, an average/peak power consumption of 1006/1127 kW, and an average/peak telemetry rate of $76 / 1800 \mathrm{kbps}$. A significant challenge of the study was to design a spacecraft with the mass and volume to accommodate the $9.5 \mathrm{~m}$ focal length that would fit within a Falcon 9 fairing that could be delivered to an L2 orbit. Key mission parameters for $N-C A L$ and the other three missions studies are contained in Table 6.

Table 6: Key Parameters of Notional Missions

\begin{tabular}{|l|r|r|r|r|}
\hline & \multicolumn{1}{|c|}{ N-CAL } & \multicolumn{1}{|c|}{ N-XGS } & \multicolumn{1}{|c|}{ N-WFI } & \multicolumn{1}{|c|}{ AXSIO } \\
\hline $\begin{array}{l}\text { Mission lifetime } \\
\text { (yrs) }\end{array}$ & 3 & 3 & 3 & 3 \\
\hline Orbit & L2 halo & L2 halo & L2 halo & L2 halo \\
\hline Mass (kg) & 1775 & 828 & 2344 & 2987 \\
\hline $\begin{array}{l}\text { Average/peak } \\
\text { power (W) }\end{array}$ & $1006 / 1127$ & $646 / 1451$ & $942 / 1629$ & $2482 / 3807$ \\
\hline $\begin{array}{l}\text { Average/peak } \\
\text { data rate (kbps) }\end{array}$ & $76 / 1800$ & $64 / 640$ & $44 / 354$ & $197 / 2125$ \\
\hline Launch vehicle & Falcon 9 & Falcon 9 & Atlas V-411 & Atlas V-511
\end{tabular}

The point design cost estimate for the $N$-CAL mission, determined from the MDL study and assuming all technology is at TRL-6 or higher, is $\$ 1.2 \mathrm{~B}$. The cost estimates for $N-C A L$ and the other three notional missions are summarized in Table 7.

\subsection{Notional Grating Spectrometer Mission ( $N$-XGS)}

The second notional mission addresses the $I X O$ science made possible by its X-ray Grating Spectrometer (XGS). Intended to be a moderate cost observatory $(\leq \$ 600 \mathrm{M})$ dedicated to high-resolution soft $\mathrm{X}$-ray grating spectroscopy, $\mathrm{N}$ XGS would provide effective area of $450 \mathrm{~cm}^{2}$ in the $0.3-1.0 \mathrm{keV}$ band, a spectral resolving power $\lambda / \Delta \lambda>3000$, and a spectroscopic capability far beyond current missions (Fig.3). $N$-XGS would explore the evolution of cosmic structure with unprecedented sensitivity to physical conditions in the WHIM, detecting absorption features with equivalent width as low as $5 \mathrm{~m} \AA$ (at $5 \sigma$ significance in $<500 \mathrm{ks}$ exposure) in $\sim 50$ blazars. Deeper exposures of the brightest of these objects would be sufficiently sensitive to detect superwind-driven shells of outflowing matter around intervening galaxies. It would probe both the velocity and density, and thus, crucially, the mass outflows fed back from supermassive black holes to their host galaxies. $N$-XGS would look for atmospheric absorption features in the spectra of bursting neutron stars. If detected, these would constrain the mass and radius, and thus the equation of state in these super-dense objects, via gravitational redshift and pressure broadening effects. $N$-XGS would bring major advances to 
the study of stars, star formation and associated flows of matter. It would resolve the thermal line widths of coronal plasmas, distinguish the kinematics of and physical conditions in accreting and outflowing material, and characterize their angular momentum, disk irradiation and magnetic dynamos.
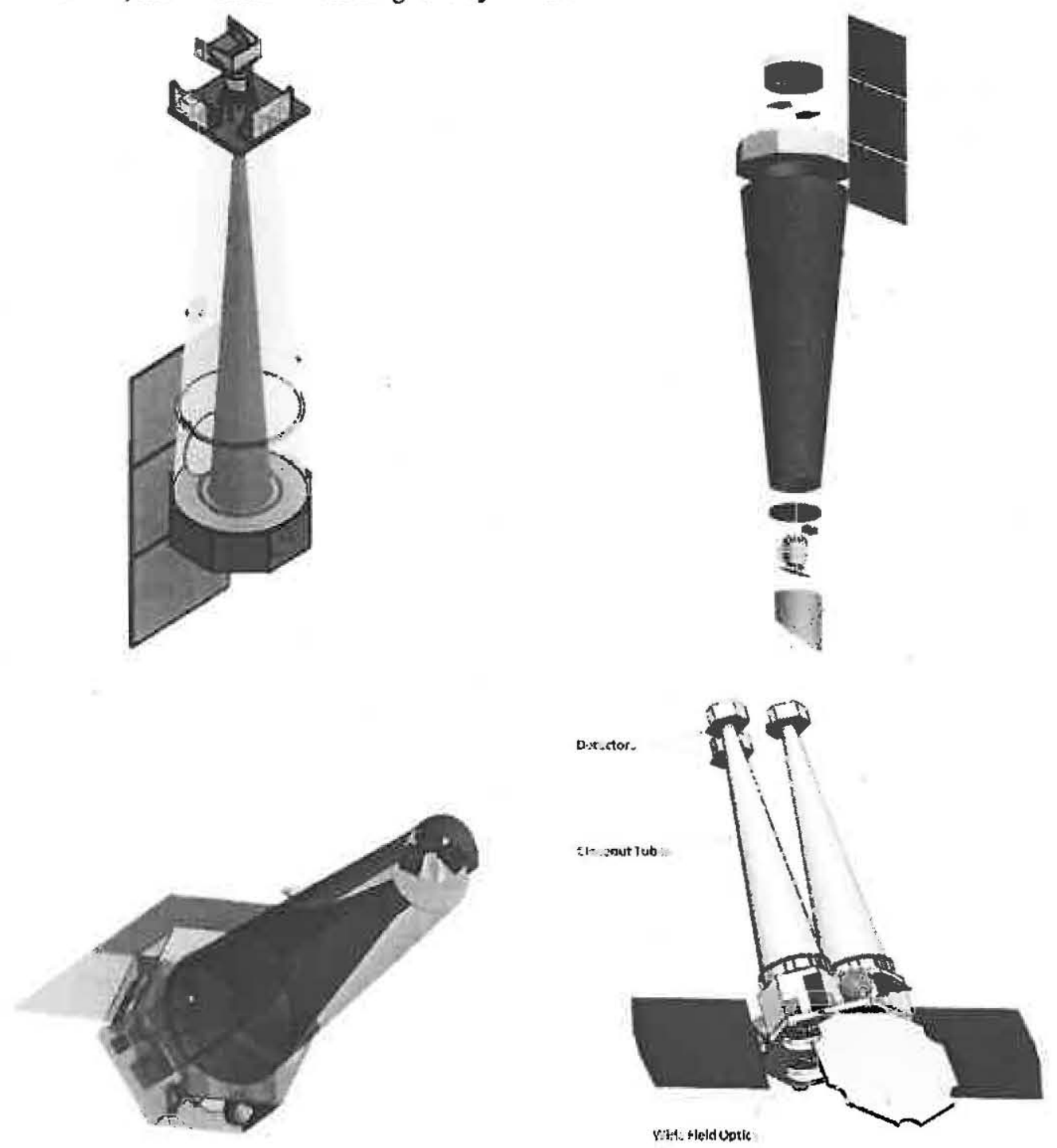

Fig. 2: Conceptual drawings of the four notional missions, from the GSFC MDL studies. The drawings are not to scale. Top left: $N$-CAL, with a $9.5 \mathrm{~m}$ focal length. Top right: $A X S I O$, with a $10 \mathrm{~m}$ focal length. Bottom left: $N$-XGS, with a $4.5 \mathrm{~m}$ focal length. Bottom right: $N$-WFI, with a $6 \mathrm{~m}$ focal length.

\subsection{1 $N$-XGS Instrumentation}

The $N$-XGS consists of two independent, objective grating spectrometers that operate in parallel. Each spectrometer is served by a set of four grazing-incidence mirror modules, which can be thought of as azimuthal sub-apertures of a circular mirror (Fig. 4). Each module produces an astigmatic image, and the optical design maximizes spectral resolving power by dispersing the spectrum from each module separately and parallel to the narrow dimension of its image. The Line-Spread Function (LSF, characterized by its full-width at half-maximum, FWHM) is roughly a factor of three smaller than the half-power diameter of the full-aperture mirror ( $10 \operatorname{arcsec})$. For $N-X G S$, a LSF with FWHM $<3$ arcsec is sufficient to achieve the required spectral resolving power $(\lambda / \Delta \lambda>3000)$. The astigmatic image produced by each $15^{\circ}$ 
module of the flight mirror assembly (FMA) meets this requirement.

Table 7: Notional Mission Cost Estimate Summary

\begin{tabular}{|l|l|c|c|c|c}
\hline WBS & & $\begin{array}{c}\text { N-CAL } \\
\text { \$M(FY12) }\end{array}$ & $\begin{array}{c}\text { N-XGS } \\
\text { \$M(FY12) }\end{array}$ & $\begin{array}{c}\text { AXSIO } \\
\text { \$M(FY12) }\end{array}$ & $\begin{array}{c}\text { N-WFI } \\
\text { \$M(FY12) }\end{array}$ \\
\hline $\mathbf{1 . 0}$ & Proj. Mgmt. & $\mathbf{s}$ & 31.6 & 66.8 & 37.1 \\
\hline $\mathbf{2 . 0}$ & Systems Eng. & 50.0 & 31.6 & 66.8 & 37.1 \\
\hline $\mathbf{3 . 0}$ & S\&MA & 31.3 & 19.7 & 41.8 & 23.2 \\
\hline $\mathbf{4 . 0}$ & Science & & & & \\
\hline & Science Team & 49.4 & 30.0 & 63.6 & 35.3 \\
\hline & GO Grants & 79.0 & 26.2 & 78.6 & 30.0 \\
\hline $\mathbf{5 . 0}$ & Payload & & & & \\
\hline & Calorimeter/WFI & 197.6 & & 197.6 & 83.3 \\
\hline & Gratings & & 101.1 & 78.0 & \\
\hline & FMA & 71.2 & 24.2 & 105.7 & 84.5 \\
\hline & FMA Facilities & 76.7 & 40.3 & 140.4 & 16.3 \\
\hline $\mathbf{6 . 0}$ & Spacecraft & 303.2 & 228.8 & 337.0 & 279.9 \\
\hline $\mathbf{7 . 0}$ & Mission Ops. & 49.1 & 50.3 & 49.1 & $\mathbf{5 0 . 3}$ \\
\hline $\mathbf{9 . 0}$ & Ground Sys. & 30.2 & 30.2 & 39.8 & 30.2 \\
\hline $\mathbf{1 0 . 0}$ & Systems I\&T & 37.5 & 23.7 & 45.9 & 27.8 \\
\hline $\mathbf{1 1 . 0}$ & E/PO & 9.8 & 6.4 & 13.1 & 7.4 \\
\hline $\mathbf{8 . 0}$ & Launcher & 140.0 & 140.0 & 230.0 & 210.0 \\
\hline & Total & $\mathbf{1 1 7 5 . 0}$ & $\mathbf{7 8 4 . 1}$ & $\mathbf{1 5 5 4 . 2}$ & $\mathbf{9 5 2 . 4}$ \\
\hline
\end{tabular}
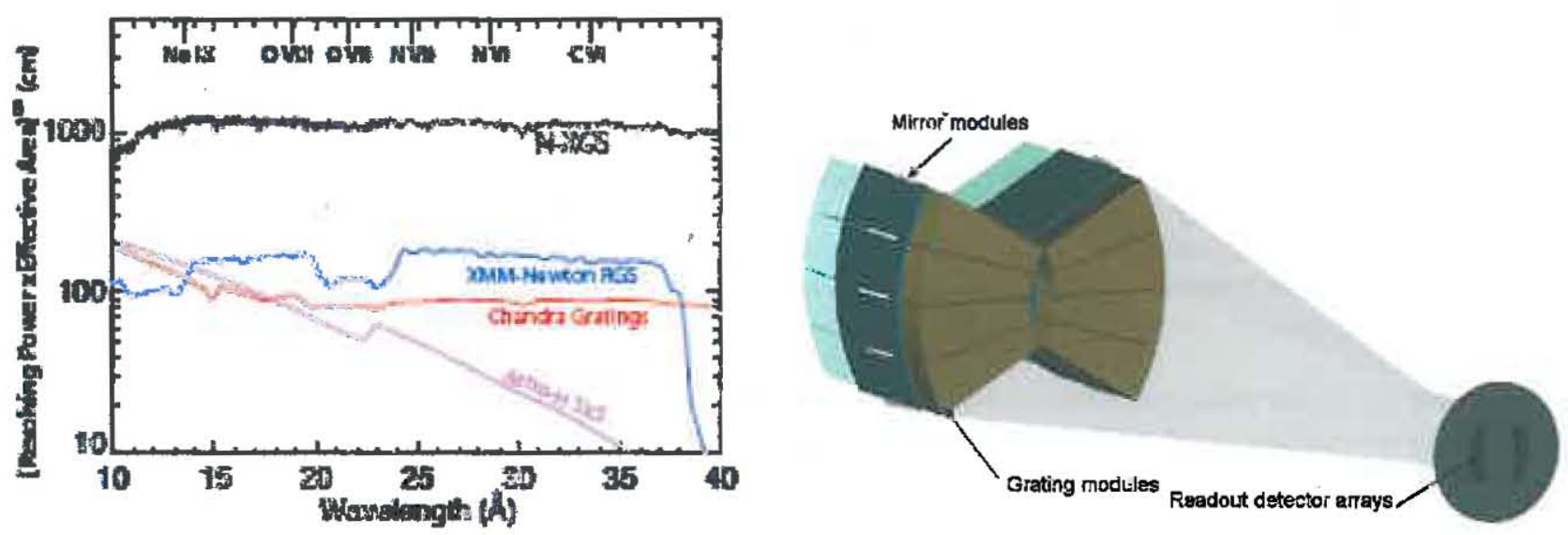

Fig 3 (left): N-XGS would provide at least a five-fold increase in sensitivity to key absorption lines (which is proportional to the square-root of the effective-area $\mathrm{x}$ resolving power product) over any current or planned spectrometer.

Fig. 4 (right): Notional XGS optical layout. Each of the two spectrometers consists of a wedge-shaped set of mirror modules spanning a $60^{\circ}$ annular sector, associated objective grating modules and a readout detector array in the focal plane.

The $N$-XGS FMA modules use the same Wolter-I, segmented glass architecture adopted for the $N$-CAL and AXSIO missions. Each $15^{\circ}$ FMA module consists of two radial sub-sectors together spanning 400 to $900 \mathrm{~mm}$ in radius. To minimize the spacecraft mass and volume, eight FMA sectors are arranged in a "bow-tie" configuration as shown in Fig. 4. 
Blazed objective gratings are mounted immediately behind each mirror module. Two distinct grating technologies are under development for the $N$-XGS: Off-Plane Gratings (OPG) ${ }^{4}$ and Critical Angle Transmission (CAT) gratings. ${ }^{5.6}$ For concreteness, the MDL study of the $N$-XGS focused on the OPG implementation, but showed that both the OPG and CAT implementations could be developed within the resource constraints deduced for the OPG version.

For either choice of grating, the dispersed spectra are detected and recorded by arrays of $\mathrm{X}$-ray photon counting, chargecoupled device (CCD) detectors. The intrinsic CCD energy resolution separates the multiple spectral orders overlapping on the focal plane. The detectors are enhanced versions of those now operating on Chandra and Suzaku.

\subsubsection{N-XGS Mission Description and Cost Estimate}

The $N$-XGS payload uses a compact, conventional spacecraft. Details of the mission are summarized in Table 6 and the spacecraft configuration is illustrated in Fig. 2. $N$-XGS is launched directly into a Sun-Earth L2 halo orbit by a Falcon-9 vehicle. This orbit maximizes observing efficiency and simplifies some aspects of spacecraft design and operations. The nominal three-year mission provides more than $70 \mathrm{Ms}$ of science exposure time, and the exposure-effective area product exceeds that baselined for IXO by a factor greater than 1.6.

The point design cost estimate for the $N$-XGS mission is $\$ 784 \mathrm{M}$. Table 7 shows the cost by WBS element, with reserves included.

\subsection{Notional Wide Field Imager Mission ( $N$-WFI)}

The third notional mission, the Wide Field Imager $(N-W F I)$, is intended as a sky survey instrument, with effective area $5000 \mathrm{~cm}^{2}$ at $1 \mathrm{keV}$ and $2,000 \mathrm{~cm}^{2}$ at $6 \mathrm{keV}, \leq 7$ arcsec angular resolution across a field of view of at least 24 arcmin, and $\mathrm{CCD}$ spectral resolution $(\sim 150 \mathrm{eV}$ at $6 \mathrm{keV})$. The $N$-WFI incorporates uses three identical telescopes, each consisting of a wide-field imaging mirror and a CCD focal plane detector. $N-W F I$ is envisioned as a $\sim \$ 1 \mathrm{~B}$ class mission.

$N$-WFI strongly addresses three $I X O$ goals. In particular, measurements of the mass and spatial distribution of clusters of galaxies to redshifts of 2 , along with the spatial distribution of AGN, would definitively address the $I X O$ science objective "How does large scale structure evolve?" By defining the luminosity function of AGN as a function of redshift (to $\mathrm{z} \sim 6$ ), notably including obscured AGN often missed by other surveys, and determining the host galaxy properties and environment, the NWFI surveys would answer the IXO questions of "When and how did supermassive black holes grow?" The large numbers of clusters and groups of galaxies that $N$-WFI would study with good angular resolution would reveal the roles of AGN outbursts and how they may change as a function of redshift which would address the IXO science objective "What is the connection between supermassive black hole formation and evolution of large scale structure (i.e., cosmic feedback)?"

Assuming a three-year mission lifetime, $N$-WFI would conduct two surveys. A medium depth survey, with an average exposure time of $20 \mathrm{ks}$, would cover about $850 \mathrm{deg}^{2}$ and take about 1.5 years (reaching a $0.5-2.0 \mathrm{keV}$ point source 50 flux limit of $\sim 4 \times 10^{-16} \mathrm{erg} \mathrm{cm}^{-2} \mathrm{~s}^{-1}$ ). A deep survey, with an average exposure time of $400 \mathrm{ks}$, would cover about $25 \mathrm{deg}^{2}$ and take about one year (reaching a point source $5 \sigma$ flux limit of $\sim 7 \times 10^{-17} \mathrm{erg} \mathrm{cm}^{-2} \mathrm{~s}^{-1}$ ). The medium survey areas would be one or two large contiguous regions to enable spatial and angular correlation studies. The deep survey would cover several smaller regions, 5-10 $\mathrm{deg}^{2}$ each, and would be spread over time in $40 \mathrm{ks}$ exposures to permit time domain studies on a variety of scales. $N-W F I$ would detect approximately $1.5 \times 10^{6} \mathrm{AGN}$ in the medium survey and an additional $2.5 \times 10^{5} \mathrm{AGN}$ in the deep survey. Of these about $1.5 \times 10^{5}$ would have more than about 400 counts, and $\sim 4,500$ would have high column density. A large number of AGN would be detected at high redshift $(\mathrm{z}>6)$ ), although predictions vary by orders of magnitude. Similarly, $N$-WFI would detect approximately 10,000 clusters in the medium survey and an additional 750 clusters in the deep survey areas. A few hundred of these would have sufficient counts to map temperature profiles; many thousands would have accurate temperature and mass estimates.

\subsubsection{N-WFI Instrumentation}

Wide field optics can be considered essentially Wolter-I but with small perturbations to their design to reduce off-axis 
aberrations and enhance resolution across a broader field of view. ${ }^{7}$ These design changes can include slight modifications to the figure of the mirror and varying the mirror shell lengths as a function of radius. The $N$-WFI design assumes full-shell fused-silica-based optics, with 71 nested shells in each of three 6-m-focal-length mirror modules. The shells range in diameter from $0.3 \mathrm{~m}$ to $0.96 \mathrm{~m}$ and vary in total reflector length (parabolic plus hyperbolic sections combined) from $300 \mathrm{~mm}$ to $480 \mathrm{~mm}$, smaller diameter mirrors being shorter in length. This design achieves the required sub-7-arcsec angular resolution out to 18 arcmin off axis and better than $7000 \mathrm{~cm}^{2}$ effective area on axis at $1 \mathrm{keV}$ and $\sim 1,800 \mathrm{~cm}^{2}$ at $6 \mathrm{keV}$ (Fig. 5). Although the HPD exceeds 7 arcsec beyond 18 arc minutes off-axis, the field of view extends to 30 arc minutes off-axis.

The detectors for the $N$-WFI mission are $2 \times 2$ arrays of X-ray CCDs. The baseline device is an MIT/Lincoln Laboratory CCD similar to those in operation on Chandra and Suzaku.
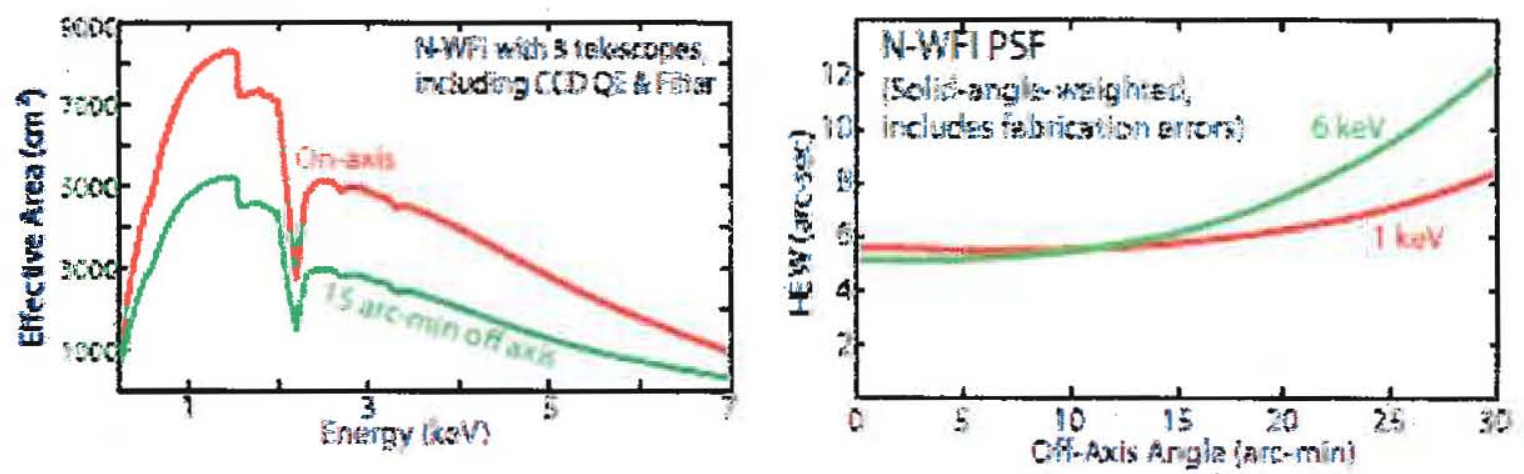

Fig. 5: Left: Net effective area versus energy for the N-WFI payload. Right: Angular resolution (solid angle weighted) versus off-axis angle for the N-WFI telescope.

\subsubsection{N-WFI Mission Description and Cost Estimate}

The three-telescope design approach allows for a compact mission (focal length of $6 \mathrm{~m}$ ) while achieving large effective area up to $6 \mathrm{keV}$. Each of the independent X-ray Telescope/Instrument assemblies includes an embedded aspect camera that provides the pointing information for that telescope. This arrangement relaxes the mechanical alignment tolerances of the spacecraft to reduce complexity and cost. The spacecraft provides the closeout tubes and optical benches for the mirrors and detectors, as well as a deployable front cover/sunshade as depicted in Fig. 2. The primary mission parameters are listed in Table 6. The total mission mass is $2344 \mathrm{~kg}$. An Atlas V 411 launch vehicle would be used to place $\mathrm{N}$-WFI into an L2 halo orbit.

The cost estimate for $N-W F I$ is broken down by WBS element, with reserves included, in Table 7. As with the other notional missions, TRL-6 is assumed for the X-ray mirrors and the focal plane detectors. The estimated total mission cost is $\$ 952 \mathrm{M}$.

\subsection{Advanced X-ray Spectroscopic and Imaging Observatory (AXSIO)}

Following the dissolution of the $I X O$ mission and prior to the commencement of this study, the $I X O$ team undertook a study of a reduced mission that would address $I X O$ science goals within the $N W N H$ cost recommendation of $<\$ 2 \mathrm{~B}$. This mission, $A X S I O$, has two instruments, a calorimeter and a grating spectrometer. $A X S I O$ was reviewed again during the $\mathrm{X}$-ray mission concepts study to verify that costing and design methodologies were consistent with those employed for the other notional missions. AXSIO and its scientific objectives are described in detail elsewhere in these proceedings. ${ }^{8}$

AXSIO retains most of $I X O$ 's scientific power thanks to a combination of the calorimeter and grating spectrometer in fixed position behind a smaller mirror. AXSIO's mirror provides nearly a factor of two more area at $1 \mathrm{keV}$ than those of $N-C A L$ or $N$-XGS, and provides the same effective area at $6 \mathrm{keV}$ as $N-C A L$ 's. All three missions were designed for a three-year lifetime, most of which would be spent observing bright sources not dominated by background. Thus AXSIO 
could achieve all of both the $N$-CAL and $N$-XGS science objectives within the same three years simply using its larger effective area.

AXSIO would make significant advances on all five of the primary $I X O$ science objectives (Table 4). Unlike singleinstrument missions, however, $A X S I O$ also has unique complementary capabilities that are required to address some $I X O$ (and $N W N H$ ) goals. Two examples demonstrate these synergistic efforts. The $I X O$ science plan to address the question, "How does large scale structure evolve?" combines absorption spectroscopy using grating observations of background AGN and imaging spectroscopy of galaxy clusters. Similarly, understanding how black hole winds form and propagate requires high-resolution spectroscopy over a broad bandpass from $0.1-10 \mathrm{keV}$, capabilities only possible using both grating and calorimeter spectrometers.

\subsubsection{AXSIO Instrumentation}

AXSIO's flight mirror assembly (FMA) is based on a segmented Wolter-I design with precision slumped glass mirror segments, the same general approach as used for the $I X O, N-X G S$ and $N-C A L$ mirrors. The mirror has a $10 \mathrm{~m}$ focal length, a diameter of $1.8 \mathrm{~m}$, and consists of 227 shells, each with a length of $40 \mathrm{~cm}(\mathrm{P}+\mathrm{H})$. The AXSIO focus on spectroscopic science allows the angular resolution requirement to be relaxed to $10 \mathrm{arcsec}$; still over an order of magnitude improvement over Astro-H. The mirror effective area is shown in Fig. 6, this FMA provides $0.9 \mathrm{~m}^{2}$ at $1 \mathrm{keV}$ (about a factor of two larger than the $N-C A L$ mirror) and $0.2 \mathrm{~m}^{2}$ at $6 \mathrm{keV}$ (essentially the same as the $N-C A L$ mirror).

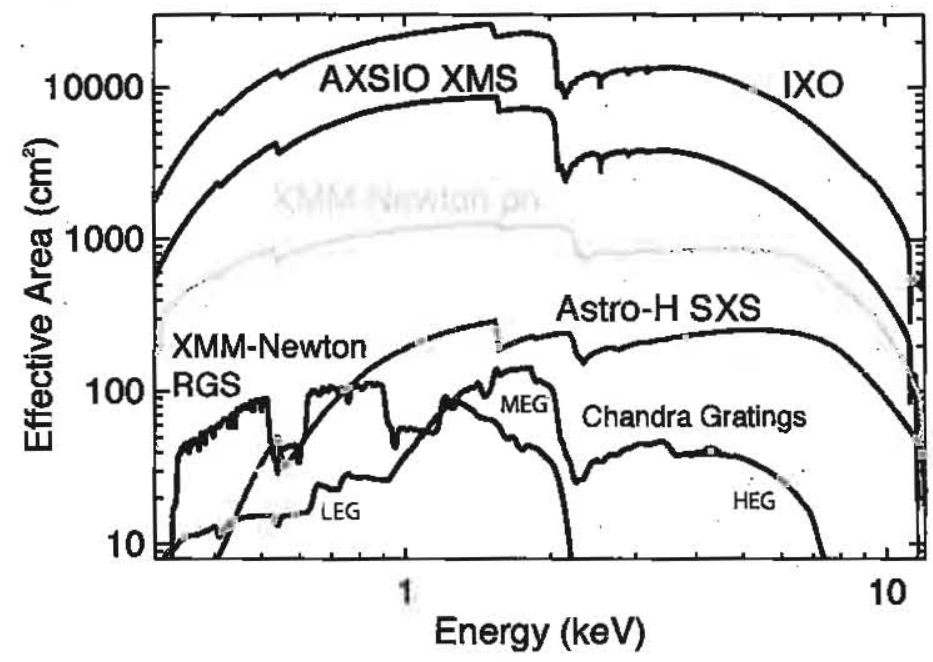

Fig 6: Comparison of AXSIO effective area with those of IXO, Astro-H, and currently operating missions.

AXSIO combines two instruments, an X-ray calorimeter spectrometer with an X-ray grating spectrometer. $A X S I O$ 's calorimeter is identical to that of $N-C A L$ : a hybrid array that includes a small central point source array (PSA) with $16 \times 16$ pixels ( 1.5 arcsec pixels) optimized for higher spectral resolution and a fast readout for studying high count-rate point sources.

AXSIO's retractable high efficiency X-ray grating spectrometer enables high-resolution spectroscopy of point sources, used either in tandem with the calorimeter or removed when observing diffuse sources. The grating spectrometer offers spectral resolution $(\lambda / \Delta \lambda)$ of 3000 (FWHM) and effective area of $1000 \mathrm{~cm}^{2}$ across the $0.3-1.0 \mathrm{keV}$ band. The CAT implementation was selected for study and costing, though the OPG is a fully viable alternative. The CAT gratings are mounted immediately behind the mirror module, with a mechanism to remove them from the beam when required.

\subsubsection{AXSIO Mission Design and Cost Estimate}

AXSIO is a facility-class observatory that would be placed via direct insertion into an L2 halo orbit; an Atlas V 521 provides substantial throw margin. Key mission parameters are shown in Table 6. The observatory's modular design is 
well cefined, building on studies performed over the last decade for Constellation- $X$ and $I X O$, and has strong heritage from previous space flight missions (Fig. 2). The L2 orbit facilitates high observational efficiency ( $>85$ percent) and provides a stable thermal and radiation environment that simplified the overall mission architecture. The MDL study concluded that the AXSIO spacecraft could be built with fully mature technologies. All subsystems utilize established hardware with substantial flight heritage.

While the original $A X S I O$ mission design life was five years, with consumables sized for 10 years, to ensure comparable costing with the notional missions this was reduced during the delta-MDL run to a three year mission with five years of consumables. As with all of the notional missions, TRL-6 is assumed for the X-ray mirrors and the focal plane detectors. The total cost for AXSIO including reserves is $\$ 1.5 \mathrm{~B}$ (see Table 7).

\section{TECHNOLOGY NEEDS FOR NOTIONAL MISSIONS}

While costing the notional missions, it was assumed that all needed technology had attained TRL-6. Most of the critical instrument technology is currently at TRL-3 or 4. RFI responses submitted for each of these areas allowed the study team to assess the timescale and cost for bringing them to TRL-6. The most critical area is optics, since it is common to all notional missions. Also critical are large calorimeter arrays, needed for $N-C A L$ and $A X S I O$, and gratings, needed for $N$-XGS and AXSIO. Longer-term technology needs, for future "vision" missions are high-resolution optics and large array active pixel detectors and fast readouts.

\section{SUMMARY}

The NASA X-ray Mission Concepts Study was performed to determine whether substantially lower cost missions could achieve the high value $I X O$ science objectives in whole or in part. Four notional mission concepts were defined, representing various nominal cost points and fulfilling as an ensemble the full range of $I X O$ science objectives. These notional mission concepts represent "point designs": substantial cost savings might be realized through optimization. Provided all needed technology were sufficiently mature (TRL-6 or higher), then the study shows that a mission fulfilling a substantial fraction of the $I X O$ scientific objectives could be developed at the cost of $\sim \$ 1 \mathrm{~B}$. If sufficient investment can be made in the critical technology areas over the next few years, then it would be possible to commence the development of such a mission before the end of the decade.

\section{REFERENCES}

[1] Bookbinder, J., "The International X-ray Observatory," arXiv:1001.2329 (2010)

[2] Barcons, X., et al., "International X-ray Observatory (IXO) Assessment Study Report for the ESA Cosmic Vision 2015-2025," arXiv:1102.2845 (2011)

[3] Blandford, R., et. al., "New Worlds, New Horizons in Astronomy and Astrophysics (NWNH)," The National Academies Press, Washington, D. C. (2010).

[4] McEntaffer, R. L., Cash, W., Lillie, C., Casement, S., Zhang, W.,; Holland, A., Murray, N., O'Dell, S., Schattenburg, M., Heilmann, R., and Tsunemi, H., "Development of off-plane gratings for WHIMex and IXO," Proc. SPIE, 8147, 81471K-81471K-11 (2011).

[5] Heilmann, R. K., Bruccoleri, A., Mukherjee, P., Yam, J., and Schattenburg, M. L., "Fabrication update on criticalangle transmission gratings for soft x-ray grating spectrometers," Proc. SPIE, 8147, 81471L-81471L-8 (2011).

[6] Heilmann, R. K., Ahn, M., Bruccoleri, A., Chang, C.-H., Gullikson, E. M., Mukherjee, P., and Schattenburg, M. L., "Diffraction efficiency of 200-nm-period critical-angle transmission gratings in the soft $\mathrm{x}$-ray and extreme ultraviolet wavelength bands," Applied Optics, 50, 1364-1373 (2011).

[7] Conconi, P., Campana, S., Tagliaferri, G., Pareschi, G., Citterio, O., Cotroneo, V., Proserpio, L., and Civitani, M., "A wide field X-ray telescope for astronomical survey purposes: from theory to practice," MNRAS, 405, 877-886 (2010).

[8] Bookbinder, J., The Advanced X-ray Spectroscopic and Imaging Observatory (AXSIO), this conference [8443-43] (2012). 